New windows on massive stars: asteroseismology, interferometry, and spectropolarimetry

Proceedings IAU Symposium No. 307, 2014

G. Meynet, C. Georgy, J. H. Groh \& Ph. Stee, eds.

(C) International Astronomical Union 2015 doi:10.1017/S1743921314006632

\title{
Wolf-Rayet stars from Very Massive Stars
}

\author{
Norhasliza Yusof \\ Department of Physics, University of Malaya, 50603 Kuala Lumpur, Malaysia \\ email: norhaslizay@um.edu.my
}

\begin{abstract}
Many studies focused on very massive stars (VMS) within the framework of Pop. III stars, because this is where they were thought to be abundant. In this work, we focus on the evolution of VMS in the local universe following the discovery of VMS in the R136 cluster in the Large Magellanic Cloud (LMC). We computed grids of VMS evolutionary tracks in the range $120-500 M_{\odot}$ with solar, LMC and Small Magellanic Cloud metallicities. All models end their lives as Wolf-Rayet (WR) stars of the WC (or WO) type. We discuss the evolution and fate of VMS around solar metallicity with particular focus on the WR phase. For example, we show that a distinctive feature that may be used to disentangle Wolf-Rayet stars originating from VMS from those originating from lower initial masses is the enhanced abundances of $\mathrm{Ne}$ and $\mathrm{Mg}$ at the surface of WC stars.
\end{abstract}

Keywords. stars: evolution, rotation, Wolf-Rayet

\section{Introduction}

In this work we discuss very massive stars (VMS) in the local Universe. Although there is high interest within Pop. III framework (Heger \& Woosley 2002; Yoon et al. 2012), evidence from observations of main sequence stars (Crowther et al. 2010) and superluminous supernovae (Gal-Yam et al. 2009) suggest that VMS exist in the local Universe. Our main interest is the evolution of VMS, in particular during their WolfRayet (WR) phases before the end of their lives. Here we discuss the WR stars originating from VMS at solar metallicity $(Z=0.014)$. As we show below, Wolf-Rayet stars from VMS have distinctive features that can be used to distinguish them from those evolving from less massive stars (less than $100 M_{\odot}$ ).

\section{Wolf Rayet stars from Very Massive Stars}

We have computed the evolution of very massive stars from $120 M_{\odot}$ to $500 M_{\odot}$ for both rotating and non-rotating models (Yusof et al. 2013). The models extend at least until the end of He-burning and, in most cases, until the start of O-burning. All the computed models evolves into WR stars, which is assumed here to happen when the surface mass fraction of hydrogen is less than 0.3 and when the effective temperature is greater then $10^{4} \mathrm{~K}$. In this present work, VMS go through the following phases; O-late WN- early WN $-\mathrm{WC} / \mathrm{WO}$. This is similar to the $M_{\mathrm{OWR}}$ evolution scenario reported in Meynet \& Maeder (2005).

In Fig. 1 we present the evolution of the surface abundances of 60 and $150 M_{\odot}$ models as a function of total mass. From the figure, one can observed that mass loss effects and internal mixing change the models surface composition. Qualitatively, there are very small differences between the 60 and $150 M_{\odot}$ models. In the $150 M_{\odot}$ model, the star has larger cores, and the transition to the various WR stages occurs at higher total masses compared to the $60 M_{\odot}$ model. This confirms the general idea that a more massive WR star originates from a more massive O-type star. Figure 1 also shows that all surface 

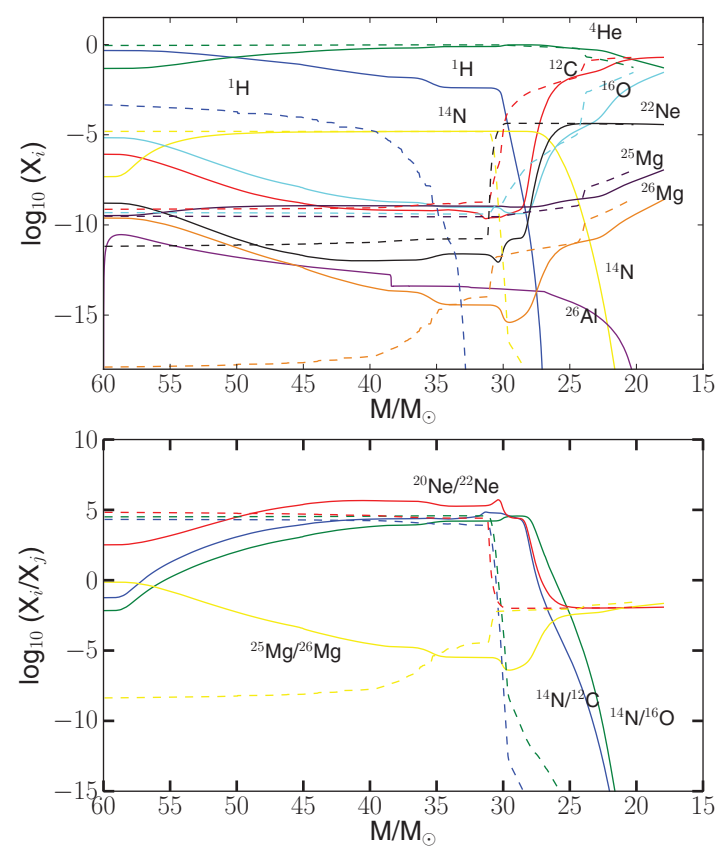

Figure 1. Top: Surface abundances of $150 M_{\odot}$ (dashed) and $60 M_{\odot}$ models at solar metallicity (dotted) as a function of the total mass. Bottom: Idem, but for surface abundance ratios.

abundances and abundance ratios are very similar for a given WR phase. It is therefore not possible to distinguish a WR originating from a VMS based on its surface chemical composition.

Although it is difficult to distinguish WRs originating from massive stars to those from VMS, we can examine the structure at the final timestep of these models. From our analysis, we find that VMS produce higher ${ }^{20} \mathrm{Ne}$ abundance. This is due to higher central temperatures during the core He-burning phase, when the reaction ${ }^{16} \mathrm{O}(\alpha, \gamma){ }^{20} \mathrm{Ne}$ is more active and, thus, more ${ }^{20} \mathrm{Ne}$ is produced. Producing large amounts of ${ }^{20} \mathrm{Ne}$ implies also enhanced ${ }^{24} \mathrm{Mg}$. We obtain layers rich in ${ }^{20} \mathrm{Ne}$ and ${ }^{24} \mathrm{Mg}$ in the $500 \mathrm{M}_{\odot}$ model. This implies that strong overabundances of these two isotopes at the surface of WC stars can be taken as a signature for an initially very massive stars as the progenitor of that WC star.

\section{Acknowledgements}

N. Yusof acknowledge support from the Fundamental Research Grant Scheme (FRGS/ FP003-2013A) under Ministry of Education of Malaysia and IAU for travel grant.

\section{References}

Crowther, P. A., Schnurr, O., Hirschi, R., et al. 2010, MNRAS 408, 731

Gal-Yam, A., Mazzali, P., \& Ofek, E. O. 2009, Nature 462, 624

Heger, A. \& Woosley, S. E. 2002, ApJ 567, 532

Meynet, G. \& Maeder, A. 2005, A\& $A$ 429, 581

Yoon, S.-C., Dierks, A., \& Langer, N. 2012, A\& A 542, A113

Yusof, N., Hirschi, R., Meynet, G., et al. 2013, MNRAS 433, 1114 